\title{
Guía de Aplicaciones para Implementar Televisión por Protocolo de Internet (IPTV) en las Organizaciones de Cartagena, Colombia
}

\author{
Raúl J. Martelo, Roime Y. Orozco, Plinio Puello \\ Univ. de Cartagena, Facultad de Ingeniería, Grupo de Investigación en tecnologías de las Comunicaciones \\ e Informática, GIMATICA, Avenida del Consulado, Calle 30, No 48 - 152, Cartagena - Colombia. \\ (e-mail: rmartelog1@unicartagena.edu.co, rorozcoh25@gmail.com, ppuellom@unicartagena.edu.co).
}

Recibido Nov. 5, 2014; Aceptado Ene. 9, 2015; Versión final Feb. 27, 2015, Publicado Ago. 2015

\section{Resumen}

Se ha realizado un estudio para implementar Televisión por Protocolo de Internet (IPTV) en las organizaciones de Cartagena en Colombia. Se identificaron las tecnologías de redes de amplia cobertura (WAN, wide area network) utilizadas en la ciudad. Luego se diseñaron e implementaron topologías de red correspondientes a las WAN identificadas y se realizaron evaluaciones técnicas aplicando calidad de servicio. Por último, se elaboró la guía que brinda información sobre las aplicaciones que ofrece IPTV bajo la tecnología WAN idónea. Esto facilita el trabajo de selección de este servicio por parte de las organizaciones que están pensando incluirlo como parte de su desarrollo tecnológico.

Palabras clave: IPTV, redes de amplia cobertura, WAN, ADSL, televisión

\section{Application Guide for Implementing Internet Protocol Television (IPTV) in Organizations of Cartagena, Colombia}

\begin{abstract}
A study to implement Internet Protocol Television (IPTV) in Organizations of Cartagena ion Colombia has been done. The wide area network technologies (WAN) used in the city were identified. After that, network topologies corresponding to the identified WAN were designed and implemented and technical evaluations were performed using quality of service. Finally, a guide that provides information about the applications that include IPTV under the suitable WAN technology was elaborated. This facilitates the selection of this type of service for those organizations that are planning to incorporate it as part of their technological development.
\end{abstract}

Keywords: IPTV, wide area network, WAN, ADSL, television 


\section{INTRODUCCIÓN}

El avance de las tecnologías y telecomunicaciones genera invención de servicios y aplicaciones, que ofrecen beneficios a consumidores para acceder a contenidos de televisión y vídeo a través de redes y dispositivos de banda ancha fija y móvil (Lee et al., 2015). Actualmente, se discute sobre la posibilidad de implementar servicios y contenidos basados en televisión digital, con el fin de introducir un componente adecuado de interactividad entre el usuario y el proveedor, siendo ésta la principal diferencia de los medios tradicionales (Kim y Lee, 2013). En Colombia, no están exentos de esta tendencia y se han realizado trabajos de investigación sobre servicios que contribuyan al progreso, mejoramiento de procesos y continuidad de empresas, IPTV es uno de estos servicios. IPTV ofrece servicios multimedia como la televisión, video, audio, texto, gráficos y datos a través de redes privadas basadas en IP (Reina et al., 2011). Los operadores de red están compitiendo cada vez más con las ofertas de triple play de proveedores de cable, tanto para clientes en general como para clientes de telecomunicaciones, hasta llegar al punto de invertir en plataformas de IPTV y lanzar sus propios servicios (Fredebeul-Krein y Steingröver, 2014). La implementación de este servicio en Colombia aún no se ha posicionado de forma amplia y definitiva (Tabares y Torres, 2012) y se han realizado diversas investigaciones para contribuir a la implementación del mismo, tales como: la situación actual de IPTV y consideraciones técnicas para su migración a IPV6 (Torres et al., 2011), estudio de viabilidad para la implementación del servicio de televisión utilizando el protocolo IP (Chio y Corredor, 2008) y análisis del impacto tecnológico, legal y económico de IPTV en las condiciones actuales del mercado colombiano (Tabares et al, 2012).

Sin embargo, a pesar de los trabajos realizados, ninguno se ha enfocado directamente con un estudio para implementar IPTV en Cartagena. Un estudio más relacionado es uno sobre viabilidad para la implementación de servicios IPTV en la Universidad de Cartagena (Alcalá y Martelo, 2014) haciendo énfasis en una organización de carácter educativo. Además se centralizó en realizar recomendaciones sobre los equipos necesarios para la disponibilidad del servicio en la institución educativa, teniendo en cuenta la infraestructura tecnológica que posee. Por ende, brindar una guía de tecnologías y aplicaciones que ofrezca IPTV a las organizaciones de Cartagena es el objetivo principal de este artículo, enfocado a organizaciones del sector industrial, educativo, turístico, entre otras, que desconocen dicho servicio. Con el fin de validar la efectividad de la misma, se realizaron evaluaciones técnicas del servicio sobre las tecnologías WAN identificadas en la ciudad, al hacer énfasis en la calidad del servicio. Como conclusión final se realiza una guía donde se recomiendan aplicaciones idóneas bajo tecnologías WAN identificadas, lo que permite a organizaciones interesadas en implementar el servicio, poder seleccionar la opción favorable ajustada a sus necesidades.

\section{TRABAJOS RELACIONADOS}

El servicio de IPTV ha sido empleado en diversos campos a nivel mundial como solución a problemas de comunicación para organizaciones y ha despertado mayor interés por parte del sector industrial, proveedores comerciales y la comunidad investigadora (Vidal et al., 2010). Existen estudios para la implementación de este servicio. En (Wang et al., 2011) se describió un sistema de estimación de edad basado en la visión, capaz de detectar la edad de una persona, utilizando su cara frontal y diversas técnicas propuestas, como la alineación facial. Esto permite la detección del perfil del usuario IPTV y sugerir de forma automática servicios de streaming, publicidad y ajustes de configuración para proporcionar una experiencia IPTV personalizada al usuario. Por otro lado, en (Orbe, 2010) se realizó un estudio de migración de sistemas de audio y vídeo por suscripción bajo la modalidad de cable físico a IPTV con sugerencias en el ámbito regulador, describiendo la infraestructura general de una red IPTV y mostrando los servicios y aplicaciones que proporciona, para establecer diferencias con sistemas de cable convencionales y mostrar los factores que influyen en la migración de sistemas de cable a IPTV. En (Bikfalvi et al., 2011) se realizó una comparación entre IPTV y P2P (peer to peer - red entre pares).

La comparación se centró en problemas de utilización de ancho de banda, calidad de vídeo y escalabilidad, cuyos resultados mostraron que la transmisión multicast es más eficiente y P2P es una alternativa para transmisión de contenidos no populares con un bajo número de espectadores. En el mismo sentido, en (Song et al., 2009) se realizó un análisis total del servicio IPTV, estudiando el desarrollo de modelos de negocios y proponiendo estrategias de marketing para mejorar la satisfacción del cliente y tener éxito en la competencia del mercado para proveedores del servicio. Por su parte (Xiao et al., 2007) estudió la calidad de servicio (QoS) que debe garantizar IPTV a usuarios finales, teniendo en cuenta tecnologías subyacentes y desafíos técnicos para proveedores del servicio. De ese mismo modo, en (Bonastre et al., 2011) se dan a conocer avances de IPTV y futuras tecnologías que posicionan a este servicio en una alternativa rentable para el consumo de televisión. Finalmente, en (Rottmann, 2010) se obtuvo un laboratorio de IPTV, como plataforma de estudio que otorga la posibilidad de ser ocupada como base, para la realización de diversas pruebas de concepto relacionadas con la entrega de este servicio, utilizando guías prácticas realizadas a 
partir de las comprobaciones efectuadas al sistema, en el cual se analizan comunicaciones de protocolos involucrados que son: SIP, DIAMETER, RTSP, RTP y RTCP.

En el ámbito de redes y telecomunicaciones del territorio colombiano también se han desarrollado trabajos que buscan implementar IPTV. En (Mendoza, 2010) se diseñó un laboratorio de IPTV, con análisis de la calidad del servicio para permitir a estudiantes de pregrado o postgrado, de la facultad de ingeniería electrónica de la Universidad Pontificia Bolivariana, conocer e interactuar con este servicio que promete reemplazar a la televisión convencional. Así mismo, en (Avellaneda et al., 2014) se evaluaron las características fundamentales en la entrega de contenidos de televisión y vídeo por demanda sobre una plataforma IPTV-IMS, utilizando el protocolo de flujo en tiempo real (RTSP) y el protocolo de transferencia de hipertexto (HTTP) a pequeña escala, lo que permite determinar cuál de éstos presenta un mejor desempeño. De otro lado, en (Ferro y Hernández, 2011) se muestran las características y ventajas que tienen los sistemas IPTV sobre las actuales plataformas de televisión, teniendo en cuenta las aplicaciones que pueden brindar, utilizando la misma infraestructura de red y la calidad de servicio al usuario final.

\section{PROPUESTA PLANTEADA}

En esta sección se presenta el modelo propuesto, para facilitar la elección de servicios y/o aplicaciones que podrían brindarse bajo el servicio de IPTV, para organizaciones. La figura1 muestra el modelo conceptual planteado:
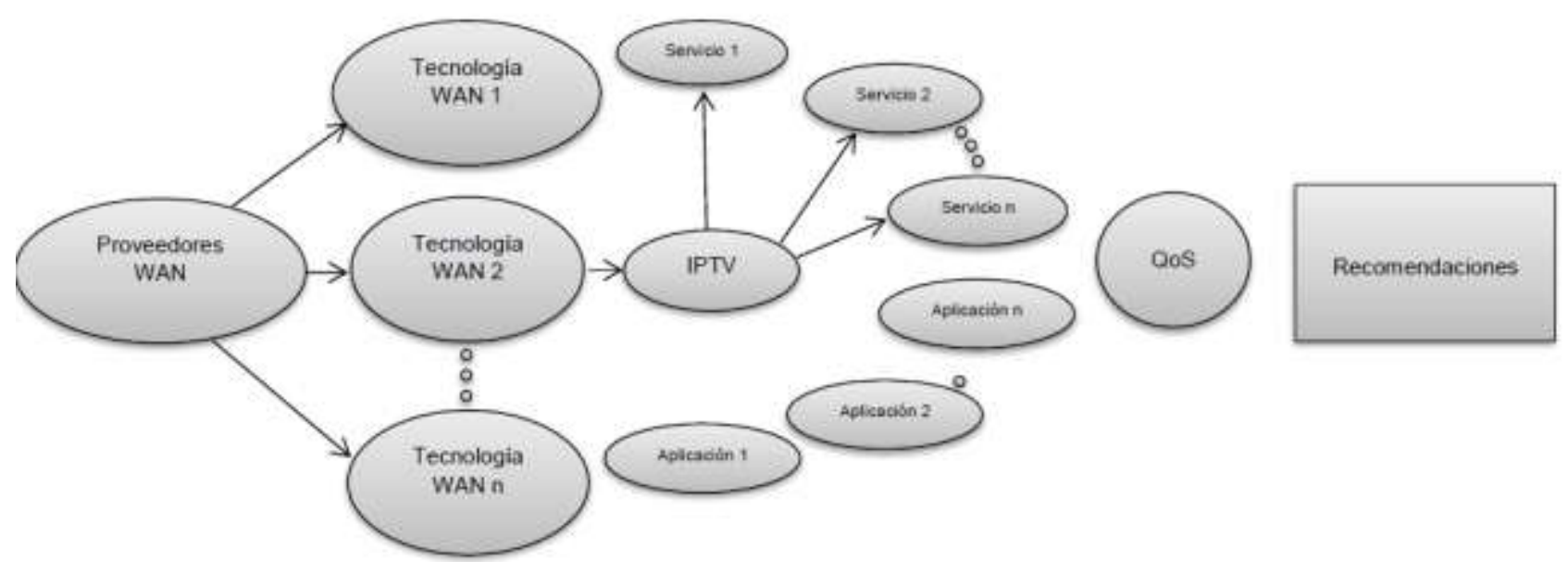

Fig. 1: Modelo conceptual planteado.

En la figura1 se pueden apreciar proveedores de servicios WAN con las tecnologías WAN que dispondría para un área geográfica determinada. Para cada tecnología, se identifican servicios y/o aplicaciones que ofrecería IPTV, luego, se les aplica calidad de servicio (QoS) para determinar cuáles son idóneas para cada tecnología WAN y ser recomendadas a organizaciones. En este modelo se visualiza la importancia de la relación que guardan los elementos, por consiguiente se realiza un estudio sobre aplicaciones que podrían utilizarse como propuesta de implementación de IPTV en las organizaciones de una o varias localizaciones geográficas. Los servicios y/o aplicaciones generados a partir del anterior modelo (figura 1) pueden ser los siguientes: E-learning, juegos en línea, pagos en línea, voto electrónico, canales corporativos, video bajo demanda $\left(V_{0} D\right)$, entre otros. Es importante resaltar que no son los únicos que ofrece IPTV.

\section{RESULTADOS}

Con el fin de validar la propuesta planteada y recomendar aplicaciones que ofrece IPTV a organizaciones, se eligió la ciudad de Cartagena como escenario. Para lograr lo anterior, se diseñaron e implementaron topologías de red sencillas sobre las tecnologías WAN ADSL y Microondas (MMDS), puesto que son ofrecidas por empresas proveedoras de internet (ISP) locales.

ADSL es utilizada por ISP en Cartagena para ofrecer servicios de telecomunicaciones a usuarios finales, dichos servicios son Telefonía IP, Internet banda ancha y el comúnmente conocido como "Triple play" que corresponde a 3 servicios en un solo paquete (Internet + Voz + Televisión), y usuarios finales son clientes residenciales o pequeñas, medianas y grandes empresas. Además, ADSL es una de las tecnologías WAN pioneras en Colombia (Muñoz et al., 2009), siendo una solución a nuevas formas de comunicación 
requeridas por clientes insatisfechos, puesto que el servicio prestado para ese entonces no era el mejor en términos de calidad y velocidad de transmisión. Por último, el mayor distribuidor de ADSL en Cartagena, Telefónica Movistar, está ofreciendo IPTV en sus nuevos paquetes de servicios, aprovechando la era de smartphones y televisores inteligentes, para ofrecer a clientes un valor agregado al producto adquirido con servicios interactivos que satisfacen las necesidades del día a día.

La necesidad de implementar nuevos servicios o de mejorar los existentes, utilizando tecnologías de fácil instalación y a su vez económicas, apoyó el surgimiento de la transmisión de datos a través de redes basadas en Microondas. Esta tecnología es utilizada como una forma rápida y económica de comunicación en el departamento de Bolívar y provee a clientes la facilidad de comunicarse con zonas dispersas geográficamente a nivel del departamento. Pequeños ISP en Bolívar, descendientes de las grandes compañías que operan en ciudades, utilizan Microondas para ofrecer servicios de telecomunicaciones que pueden ser temporales o a término fijo. Ejemplos de estas pequeñas compañías son Edatel, Megared y Compartel, siendo este último un proyecto del ministerio de las tecnologías de la información y comunicación (TIC) para ofrecer internet gratuito a zonas rurales de Colombia, reemplazando sus antenas satelitales por antenas transmisoras y receptoras basadas en Microondas. Por otro lado, Edatel es una empresa descendiente de UNE, encargada de ofrecer servicios de internet en municipios de Bolívar a través de Microondas y tiene proyectado expandir sus redes para ofrecer servicios a la zona norte del país. Por último, Cartagena es una ciudad compuesta por antenas transmisoras o receptoras de Microondas, las cuales están instaladas a la altura de las antenas para redes móviles o en plataformas superiores de edificios. Un caso puntual es el Servicio Nacional de Aprendizaje SENA, una institución de carácter público en Colombia, el cual utiliza Microondas para realizar videoconferencias con los centros de formación situados en la ciudad y en los municipios de Bolívar.

Para garantizar que las aplicaciones seleccionadas prestan mejor servicio, se realizaron pruebas para medir la calidad de imagen, paquetes transmitidos, tasas de transmisión, atenuación, ruido, BER (Bit Error Rate, Tasa de Error de Bit) y MER (Modulation Error Ratio o Relación de Error de la Modulación). Estos parámetros fueron analizados para las dos tecnologías (ADSL y Microondas), en ambos casos se capturaron frecuencias recibidas por el satélite Hispasat y se tomaron 10 muestras cada 2 minutos. La tabla 1 muestra los resultados obtenidos durante la prueba de ADSL.

Tabla 1: Datos obtenidos durante prueba de ADSL.

\begin{tabular}{|c|c|c|c|}
\hline Frecuencia 1285.13 & Unidades & Valor Obtenido & Valor Óptimo \\
\hline VBER & Adimensional & $8.0 \mathrm{E}-8$ & $1.0 \mathrm{E}-7$ hasta 2.0E-4 \\
\hline CBER & Adimensional & $2.9 \mathrm{E}-3-3.3 \mathrm{E}-2$ & $1.0 \mathrm{E}-2$ hasta 1.0E-4 \\
\hline MER & $\mathrm{dB}$ & $9.2-9.4$ & $>=21$ \\
\hline Ruido & $\mathrm{dB}$ & $9.9-10.4$ & $>=25$ \\
\hline Atenuación & $\mathrm{dB}$ & $65.4-65.9$ & $45-70$ \\
\hline
\end{tabular}

De acuerdo a la tabla 1 se concluye, que durante la prueba los valores encontrados se mantuvieron dentro de los límites establecidos para cada variable estudiada, contribuyendo a que la calidad de la señal fuera mejor. La fluctuación entre valores pudo deberse a variaciones atmosféricas en un tiempo determinado. Sin embargo, la calidad de imagen fue buena durante la prueba. La tabla 2 muestra los resultados obtenidos durante la prueba de Microondas.

Tabla 2: Datos obtenidos durante prueba de Microondas.

\begin{tabular}{|c|c|c|c|}
\hline Frecuencia 1453.09 & Unidades & Valor Obtenido & Valor Óptimo \\
\hline VBER & Adimensional & $5.8 \mathrm{E}-7-1.1 \mathrm{E}-6$ & $1.0 \mathrm{E}-7$ hasta $2.0 \mathrm{E}-4$ \\
\hline CBER & Adimensional & $1.2 \mathrm{E}-3-1.4 \mathrm{E}-2$ & $1.0 \mathrm{E}-2$ hasta $1.0 \mathrm{E}-4$ \\
\hline MER & $\mathrm{dB}$ & $7.6-7.8$ & $>=21$ \\
\hline Ruido & $\mathrm{dB}$ & $9.7-10.1$ & $>=25$ \\
\hline Atenuación & $\mathrm{dB}$ & $61.9-62.3$ & $45-70$ \\
\hline
\end{tabular}

De acuerdo a la tabla anterior se concluye, que los valores encontrados durante la prueba se mantuvieron dentro de los límites establecidos, a pesar de las variaciones que se presentaron. Dichas variaciones implicaron en que la calidad de la señal no fuera la mejor y presentara distorsiones en instantes de tiempo, 
provocando una calidad de imagen regular durante la prueba. Como se aprecia en la figura 1, la investigación fue planificada para determinar aplicaciones de IPTV que mejor se acoplen a intereses y condiciones económicas de organizaciones en Cartagena, enfocándose en los servicios que ofrecen y las tecnologías que emplea. De esta forma, la facilidad de elección se brinda a través de una guía de recomendaciones que contiene las aplicaciones que cumplen con los requerimientos encontrados.

Con el fin de dar cumplimiento al objetivo principal de este artículo, se elaboró una guía que contiene aplicaciones de IPTV recomendadas, sectores que constituyen la ciudad de Cartagena y tecnologías WAN identificadas en dicha ciudad. Dicha guía muestra a las organizaciones, una lista de aplicaciones que se pueden implementar con la tecnología WAN ajustada a sus procesos de comunicación, lo que permite que tengan opciones que contribuyen a la permanencia en el mercado, mejoramiento de procesos, toma de decisiones, servicio y atención a clientes, entre otras; teniendo en cuenta sus recursos económicos. La tabla 3 muestra los sectores de Cartagena donde IPTV tiene mayor uso y las aplicaciones recomendadas bajo las tecnologías WAN idóneas según el sector.

Tabla 3: Aplicaciones que ofrecería IPTV en organizaciones de Cartagena.

\begin{tabular}{|l|c|c|c|c|c|c|c|c|c|c|c|c|}
\hline \multicolumn{2}{|c|}{ Sector $\rightarrow$} & \multicolumn{2}{c|}{ Comercial } & \multicolumn{2}{c|}{ Educativo } & Gubernamental & \multicolumn{2}{c|}{ Industrial } & \multicolumn{2}{c|}{ ISP } & \multicolumn{2}{c|}{ Turístico } \\
\hline Aplicaciones $\downarrow$ & ADSL & MMDS & ADSL & MMDS & ADSL & MMDS & ADSL & MMDS & ADSL & MMDS & ADSL & MMDS \\
\hline Canales corporativos & $\mathrm{x}$ & $\mathrm{x}$ & $\mathrm{x}$ & $\mathrm{x}$ & $\mathrm{x}$ & $\mathrm{x}$ & $\mathrm{x}$ & $\mathrm{x}$ & $\mathrm{x}$ & $\mathrm{x}$ & $\mathrm{x}$ \\
\hline Contenidos en línea & $\mathrm{x}$ & $\mathrm{x}$ & $\mathrm{x}$ & $\mathrm{x}$ & $\mathrm{x}$ & $\mathrm{x}$ & $\mathrm{x}$ & $\mathrm{x}$ & $\mathrm{x}$ & $\mathrm{x}$ & $\mathrm{x}$ & $\mathrm{x}$ \\
\hline e-learning & & & $\mathrm{x}$ & $\mathrm{x}$ & & & & & & & & \\
\hline Juegos en línea & $\mathrm{x}$ & & & & & & & & $\mathrm{x}$ & & $\mathrm{x}$ & \\
\hline Mosaicos & & & & & & & & & $\mathrm{x}$ & $\mathrm{x}$ & $\mathrm{x}$ & \\
\hline Pagos en línea & $\mathrm{x}$ & $\mathrm{x}$ & & & $\mathrm{x}$ & $\mathrm{x}$ & $\mathrm{x}$ & $\mathrm{x}$ & $\mathrm{x}$ & $\mathrm{x}$ & $\mathrm{x}$ & $\mathrm{x}$ \\
\hline Picture in picture & & & & & & & & & $\mathrm{x}$ & & $\mathrm{x}$ & \\
\hline Publicidad interactiva & $\mathrm{x}$ & $\mathrm{x}$ & $\mathrm{x}$ & $\mathrm{x}$ & & & & & $\mathrm{x}$ & $\mathrm{x}$ & & \\
\hline PVR & & & & & $\mathrm{x}$ & & $\mathrm{x}$ & & $\mathrm{x}$ & & $\mathrm{x}$ & \\
\hline Triple play & & & & & & & & & $\mathrm{x}$ & & & \\
\hline TwitTV & $\mathrm{x}$ & $\mathrm{x}$ & $\mathrm{x}$ & $\mathrm{x}$ & $\mathrm{x}$ & $\mathrm{x}$ & $\mathrm{x}$ & $\mathrm{x}$ & & & $\mathrm{x}$ & $\mathrm{x}$ \\
\hline Videoconferencias & $\mathrm{x}$ & $\mathrm{x}$ & $\mathrm{x}$ & $\mathrm{x}$ & $\mathrm{x}$ & $\mathrm{x}$ & $\mathrm{x}$ & $\mathrm{x}$ & $\mathrm{x}$ & $\mathrm{x}$ & & $\mathrm{x}$ \\
\hline VOD & & & & & & & & & $\mathrm{x}$ & & $\mathrm{x}$ & \\
\hline Voto electrónico & & & & & $\mathrm{x}$ & $\mathrm{x}$ & & & & & & \\
\hline
\end{tabular}

De acuerdo a la tabla 3 se puede recomendar a una organización del sector turístico que para aplicaciones VOD (video bajo demanda) utilice la tecnología ADSL, mientras que para videoconferencias lo recomendable es Microondas. En el mismo sentido, para una organización del sector educativo es recomendable utilizar ADSL y Microondas para aplicaciones de e-learning, siendo opcional qué tecnología escoger a partir de sus condiciones económicas.

En el ámbito de redes y telecomunicaciones existen diversos factores externos que interfieren en una comunicación, los cuales inciden para que ésta se vea afectada en instantes de tiempo, y en ocasiones provocan inconvenientes difíciles de solucionar inmediatamente. Por tal motivo, es importante que la calidad de servicio ofrecida por servicios y/o tecnologías tenga buen rendimiento. Las aplicaciones que ofrece IPTV bajo ADSL son variadas y aumentarán con el pasar del tiempo, debido a que la calidad de servicio de IPTV en ella es buena y tiene la virtud de ser una red basada en cableado estructurado, clarificando la ventaja en calidad de servicio. Una desventaja es la instalación, en ocasiones, es un proceso riguroso que implica reestructuración de diseños de redes establecidos.

Con la tecnología microondas, IPTV ofrece aplicaciones similares a ADSL, pero al ser una red inalámbrica a gran escala, la interferencia es inevitable y puede afectar la calidad del servicio debido a que es una red basada en ondas electromagnéticas que viajan en el aire. Por ejemplo, Microondas sería esencial para una comunicación entre sucursales a través de un canal corporativo. Sin embargo, microondas tiene la virtud de fácil instalación y adaptación a infraestructuras de redes establecidas. Sin duda existen aplicaciones que son recomendadas para ambas tecnologías WAN, pues en términos generales, la aplicación funciona correctamente bajo dichas tecnologías. Sin embargo, hay puntos claves que juegan un papel importante en términos de calidad, implementación y adaptación, uno de ellos es la calidad del servicio. Por último y no menos importante, están los costos, los cuales en términos de organizaciones hacen referencia a egresos 
que se deben ver reenumerados para la continuidad y progreso de las mismas. En este aspecto, ADSL lleva la ventaja con respecto a microondas para implementar IPTV, no obstante, hay que tener en cuenta los costos adicionales, que pueden o no existir según el contexto.

Tabla 4: Tabla comparativa de recomendaciones realizadas en otros estudios.

\begin{tabular}{|c|c|c|c|c|}
\hline & Estudio & $\begin{array}{l}\text { Tecnología } \\
\text { WAN }\end{array}$ & $\begin{array}{l}\text { Aplicaciones } \\
\text { Recomendadas }\end{array}$ & Resultados \\
\hline$\overline{\mathscr{J}}$ & $\begin{array}{l}\text { Estudio de viabilidad para la para la } \\
\text { implementación de servicios IPTV en la } \\
\text { Universidad de Cartagena (Alcalá y } \\
\text { Martelo, 2014). }\end{array}$ & ADSL & VoD, videoconferencias. & Esperables. \\
\hline \multirow{3}{*}{$\begin{array}{l}\overline{\widetilde{T}} \\
\frac{\mathrm{C}}{\mathrm{O}} \\
\frac{\mathbb{T}}{Z}\end{array}$} & $\begin{array}{l}\text { Análisis de rendimiento de la transmisión } \\
\text { de IPTV sobre ADSL, WiFi y LAN } \\
\text { Extended (Nuñez et al., 2008). }\end{array}$ & $\begin{array}{l}\text { ADSL y } \\
\text { Microondas }\end{array}$ & $\begin{array}{l}\text { VoD, juegos en línea, PVR, } \\
\text { videoconferencias. }\end{array}$ & Esperables. \\
\hline & $\begin{array}{l}\text { Calidad de servicio en proveedores de } \\
\text { servicios IPTV (García y Cuellar, 2014). }\end{array}$ & ADSL & VoD, e-learning, PVR. & Esperables. \\
\hline & $\begin{array}{l}\text { Estudio de viabilidad para la } \\
\text { implementación del servicio de televisión } \\
\text { utilizando el protocolo IP en el área } \\
\text { metropolitana de Bucaramanga (Chio y } \\
\text { Corredor, 2008). }\end{array}$ & ADSL & $\begin{array}{l}\text { VoD, e-learning, PVR, } \\
\text { publicidad, triple play. }\end{array}$ & Esperables. \\
\hline \multirow{3}{*}{ 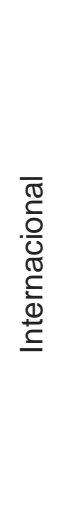 } & $\begin{array}{l}\text { La televisión IP (IPTV) y la transmisión } \\
\text { mediante VDSL: realidad y perspectivas } \\
\text { de negocio (Gascón y Francesc, 2012). }\end{array}$ & VDSL & Triple play, quadrupe play. & Mejores. \\
\hline & $\begin{array}{l}\text { Revisión de la Implementación del } \\
\text { Servicio de IPTV sobre Redes } \\
\text { Inalámbricas y Móviles con Calidad de } \\
\text { Servicio (Pepinosa y Rodríguez, 2013). }\end{array}$ & Microondas & Videoconferencias. & Peores. \\
\hline & $\begin{array}{l}\text { Estudio de una red IP/MPLS para } \\
\text { agregar servicios de televisión IP en } \\
\text { operadoras telefónicas fijas tradicionales } \\
\text { para usuarios residenciales mediante } \\
\text { tecnologías XDSL para ciudad de Quito } \\
\text { (Velásquez, 2010). }\end{array}$ & ADSL & $\begin{array}{l}\text { VoD, triple play, contenidos } \\
\text { en línea. }\end{array}$ & Esperables. \\
\hline
\end{tabular}

De acuerdo a la tabla 4, se puede apreciar que la tecnología WAN predominante en el mercado de telecomunicaciones es xDSL, teniendo mayor acogida ADSL al ser la pionera en este ámbito. Sin embargo, es considerada una tecnología ambigua que debe ser reemplazada por sus sucesores. Por otro lado, Microondas es poco usada para IPTV en otros países, debido a que cuentan con tecnologías de mejores condiciones para el servicio. Muchas recomendaciones realizadas en los estudios plasmados en la tabla 4 coinciden con las del presente estudio, sobre todo a nivel nacional y local, pues las redes WAN implementadas circulan sobre el mismo eje de telecomunicaciones lo que contribuye a que los escenarios de prueba sean similares. A nivel internacional cambian los escenarios y tecnologías utilizadas, sin embargo, las aplicaciones recomendadas son similares debido que IPTV es un servicio que ofrece una gran variedad de aplicaciones y al ser un protocolo basado en IP, la disponibilidad es constante y asequible desde cualquier lugar del mundo.

\section{CONCLUSIONES}

De los resultados obtenidos, se pueden anunciar las siguientes conclusiones sobre la propuesta: 1) Posiciona a IPTV como un servicio que era desconocido por organizaciones manejo de Cartagena; 2) Permite identificar las aplicaciones que cumplen con los requerimientos de organizaciones en Cartagena; 3 ) Apoya a la toma de decisiones en la administración y control de procesos de comunicación; 4) Es útil para innovar nuevas formas de comunicación.

\section{REFERENCIAS}

Alcalá, R. y R. J. Martelo, Estudio de viabilidad para la para la implementación de servicios IPTV en la Universidad de Cartagena. Tesis de Titulación. Facultad de Ingeniería, Universidad de Cartagena, Cartagena, Colombia (2014) 
Avellaneda, J. V., J. R. Rodríguez y D. A. López, Servicios de Televisión sobre la Plataforma de Internet (IPTV-IMS) usando Protocolo de Flujo en Tiempo Real (RTSP) y Protocolo de Transferencia de Hipertexto (HTTP), doi: 10.4067/S0718-07642014000100008, Información tecnológica: 25(1), 67-76 (2014)

Bikfalvi, A. y otros cuatro autores, P2P vs. IP multicast: Comparing approaches to IPTV streaming based on TV channel popularity, doi:10.1016/j.comnet.2010.12.020, Computer Networks, 55(6), 1310-1325 (2011)

Bonastre, O. M., M. J. Montpetit y P. Cesar, Advances in IPTV technologies, doi:10.1016/j.image.2011.05.005, Signal Processing: Image Communication, 26(7), 325-326 (2011)

Chio, N. y R. Corredor, Estudio de viabilidad para la implementación del servicio de televisión utilizando el protocolo IP en el área metropolitana de Bucaramanga. Tesis de especialización. Escuela de ingenierías y administración, Universidad Pontificia Bolivariana, Bucaramanga, Colombia (2008)

Delgado, M. y D. Fernández, IPTV: estructura de mercado y tipología de la oferta en España, http://www.ehu.es/ojs/index.php/Zer/article/view/3702/3334, ISSN: 1137-1102, Revista Zer, 12(22), 413-428 (2007)

Ferro, R.A. y C. Hernández, Los sistemas IPTV ¿una amenaza inminente para los actuales medios de teledifusión?, http://tecnura.udistrital.edu.co/ojs/index.php/revista/article/view/370/363, ISSN: 0123-921X, Revista tecnura, 15(28), 101-122 (2011)

Fredebeul-Krein, M. y M. Steingröver, Wholesale broadband access to IPTV in an NGA environment: How to deal with it from a regulatory perspective?, doi:10.1016/j.telpol.2013.04.002, Telecommunications Policy, 38(3), 264-277 (2014)

García, A.A. y J.C. Cuellar, Calidad de servicio en proveedores de servicios IPTV, http://revistas.usc.edu.co/index.php/Ingenium/article/view/91/80\#.VBDvbcJ5OSo, $\quad$ ISSN: $\quad$ 1692-0899 Ingenuim, 6(13), 11-25 (2012)

Gascón, F. y J. Francesc, La televisión IP (IPTV) y la transmisión mediante VDSL: realidad y perspectivas de negocio, http://www.ucm.es/info/vivataca/anteriores/n105/DATOSS105.htm, ISSN: 1575-2844, Vivat Academia, 11(105), 1-29 (2009)

Jácome, T. y A. Tafur, Estudio de factibilidad para la implementación del servicio de televisión IPTV en la Corporación Nacional de Telecomunicaciones (CNT). Tesis de Maestría. Vicerrectorado de investigación y vinculación con la colectividad, Universidad de las Fuerzas Armadas, Sangolquí, Ecuador (2013)

Kim, J. y K. H. Lee, Towards a theoretical framework of motivations and interactivity for using IPTV, doi:10.1016/j.jbusres.2012.07.004, Journal of Business Research, 66(2), 260-264 (2013)

Lee, S., E. A. Park, S. Lee, y J. Brown, Determinants of IPTV diffusion, doi:10.1016/j.tele.2014.10.005, Telematics and Informatics, 32(3), 439-446 (2015)

Mendoza, E., Montaje de un laboratorio de televisión sobre el protocolo IP con análisis de calidad de servicio. Tesis de Titulación. Escuela de Ingeniería y Administración, Universidad Pontificia Bolivariana, Bucaramanga, Colombia (2010)

Muñoz, J., A. Martínez y H. Paz, Tecnología iptv en Colombia, http://tecnura.udistrital.edu.co/ojs/index.php/revista/article/view/277, ISSN: 2248-7638, Revista Tecnura, 12(24), 68-75 (2009)

Núñez, C. V., J. F. Fruto y Y. D. Meisel, Análisis de rendimiento de la transmisión de IPTV sobre ADSL, WiFi y LAN Extended, http://www.scielo.org.co/pdf/inde/n23/n23a08.pdf, ISSN: 0122-3461, Ingeniería y Desarrollo, (23), 84-103 (2008)

Orbe, C., Estudio de migración de sistemas de audio y vídeo por suscripción bajo la modalidad de cable físico a IPTV con sugerencias en el ámbito regulador. Tesis de Titulación. Facultad de Ingeniería Eléctrica y Electrónica, Escuela Politécnica Nacional, Quito, Ecuador (2010) 
Pepinosa, D. F. R. y Z. I. R. Rodríguez, Revisión de la Implementación del Servicio de IPTV sobre Redes Inalámbricas y Móviles con Calidad de Servicio (QoS),

http://revistas.uis.edu.co/index.php/revistauisingenierias/article/view/3709, ISSN: 2145-8456, REVISTA UIS

Ingenierías, 12(1), 39-50 (2013)

Reina, J., D.H. Agudelo y J. C. Montoya, Análisis del retardo en el cambio de canal en IPTV, https://revistas.upb.edu.co/index.php/telecomunicaciones/article/view/1255/1147, ISSN: 0120-131X, Revista en telecomunicaciones e informática, 1(2), 61-73 (2011)

Rottmann, K., Diseño e implementación de un laboratorio de IPTV, medición y gestión. Tesis de Titulación. Departamento de Ingeniería Eléctrica, Universidad de Chile, Santiago de Chile, Chile (2010)

Song, J., T. Jang y S. Y. Sohn, Conjoint analysis for IPTV service, doi:10.1016/j.eswa.2008.11.016, Expert Systems with Applications, 36(4), 7860-7864 (2009)

Tabares, J. y J. Torres, Análisis del impacto tecnológico, legal y económico de IPTV en las condiciones actuales del mercado colombiano de las telecomunicaciones. Tesis de Titulación. Facultad de Ingenierías, Universidad Tecnológica de Pereira, Pereira, Colombia (2012)

Torres, J.I., R. E. Ramírez y D. A. López, Estado del arte de IPTV y consideraciones técnicas para su migración a ipv6 en Colombia,

http://ingenieria.udistrital.edu.co/digital/index.php/redesdeingenieria/article/view/48/119, ISSN: 2248-762X,

Redes de ingeniería, 2(1), 45-64 (2011)

Velásquez-Rivera, J. A., Estudio de una red IP/MPLS para agregar servicios de televisión IP en operadoras telefónicas fijas tradicionales para usuarios residenciales mediante tecnologías XDSL para ciudad de Quito. Tesis de titulación. Fac. Ingeniería Eléctrica y Electrónica, Escuela Politéc. Nacional, Quito, Ecuador (2010)

Vidal, I. y otros cuatro autores, Supporting mobility in an IMS-based P2P IPTV service: A proactive context transfer mechanism, doi:10.1016/j.comcom.2010.05.001, Computer Communications, 33(14), 1736-1751 (2010)

Wang, H. L., J. G. Wang y W. Y. Yau, Automated age regression for personalized IPTV services, doi:10.1016/j.image.2011.03.004, Signal Processing: Image Communication, 26(7), 390-399 (2011)

Xiao, Y. y otros cuatro autores, Internet Protocol Television (IPTV): The Killer Application for the NextGeneration Internet, doi:10.1109/MCOM.2007.4378332, Communications Magazine IEEE, 45(11), 126-134 (2007) 